\title{
K-TIPS: KNOWLEDGE EXTENSION BASED ON TAILOR-MADE INFORMATION PROVISION SYSTEM
}

\author{
Keita Nakayama, Atsushi Shimada, Tsubasa Minematsu, Yuta Taniguchi and Rin-Ichiro Taniguchi \\ Kyushu University, Japan
}

\begin{abstract}
Thanks to an increase in the amount of information on the Internet and the spread of ICT-supported educational environments, much attention has been paid to learning support based on "smart" recommendation technologies. In this study, we propose an education improvement model based on the recommender system using the human-in-the-loop design strategy. Our proposed model enhances not only learners via recommendation, but also teachers and the system itself through the interaction between teachers and the system. In this paper, we introduce the details of the proposed model and implementation strategy followed by a report of preliminary experimental results.
\end{abstract}

\section{KEYWORDS}

Education Improvement, Recommendation, Learning Analytics, Learning Log

\section{INTRODUCTION}

The increasing amount of information on the Internet, that is, the high number of open knowledge objects such as Wikipedia, iTuneU, and repositories managed by universities all over the world seems to be useful for supporting learning. However, learners are often discouraged by the time spent and difficulty in finding suitable and useful knowledge online (e.g., news articles, academic papers, and blogs) from a mass of search results (Chen et al., 2014). To use the information effectively and efficiently, it is necessary to build a system for the appropriate use of this vast knowledge. The recommender system for learning support is a common and useful system in this era of vast information (e.g., Erdt et al., 2015). Thus, a recommender system provides an appropriate learning environment that helps learners find appropriate knowledge targets within the vast amount of information on the Internet.

Researchers have been applying these resources to learning systems to clarify the effects of recommender systems for learning (e.g., Liang et al., 2006; Chen et al., 2007; Yamada et al., 2014). Many techniques for a recommender system have also been proposed. In particular, there are a large number of studies aimed at supporting learners based on student attributes such as learning situation (e.g., Tarus et al., 2018), preference based on learning logs (e.g., Salehi et al., 2014), search queries (e.g., Mbipom et al., 2018), and so on. On the other hand, there are a few studies that try, not only to support learners, but also to improve teaching materials and lectures. In addition, many existing studies unilaterally recommend online resources determined by the system, and do not design a feedback loop to improve the system itself. In order for the system to recommend appropriate resources from an educational viewpoint and for individual learners, it is desirable to involve teachers in the loop of recommendation and improvement of education and learning. In this paper, we propose an education improvement model, which collaborates with the page-wise recommendation method (Nakayama et al., 2019). The page-wise recommendation system provides recommended information for each page as shown in Figure 1. The original recommender system has some drawbacks (see the next section for the details), so our proposed model involves teachers to complement the drawbacks. In addition, the teachers can also get feedback from the system to improve their teaching and teaching materials. Therefore, our proposed model enhances not only learners via recommendation, but also the teachers and the system based on the human-in-the-loop design strategy. In the following section of this paper, we introduce the details of the proposed model and implementation strategy followed by a report of preliminary experimental results. 


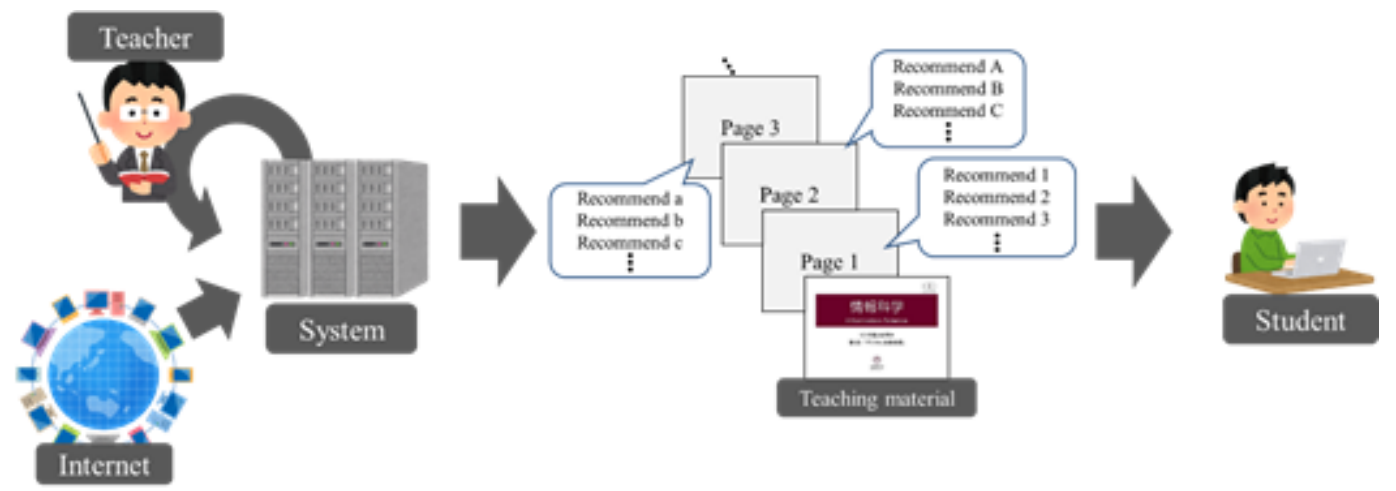

Figure 1. Page-wise recommendation system

\section{EDUCATION IMPROVEMENT MODEL BASED ON PAGE-WISE RECOMMENDATION}

In this study, we propose an education improvement model based on a system that recommends supplementary materials related to teaching materials used in lectures. The overview of the system configuration is shown in Figure 2. The proposed model improves education and learning by cycling through the following process:

(1) A teacher prepares a lecture plan and teaching material in preparation for a lecture and registers the teaching material in an e-Book system.

(2) The proposed system selects recommended information related to the teaching material from the Internet.

(3) The e-Book system provides the recommendation to the students during the lecture or self-study.

(4) The system analyzes the learning activity logs and gives feedback to the teacher.

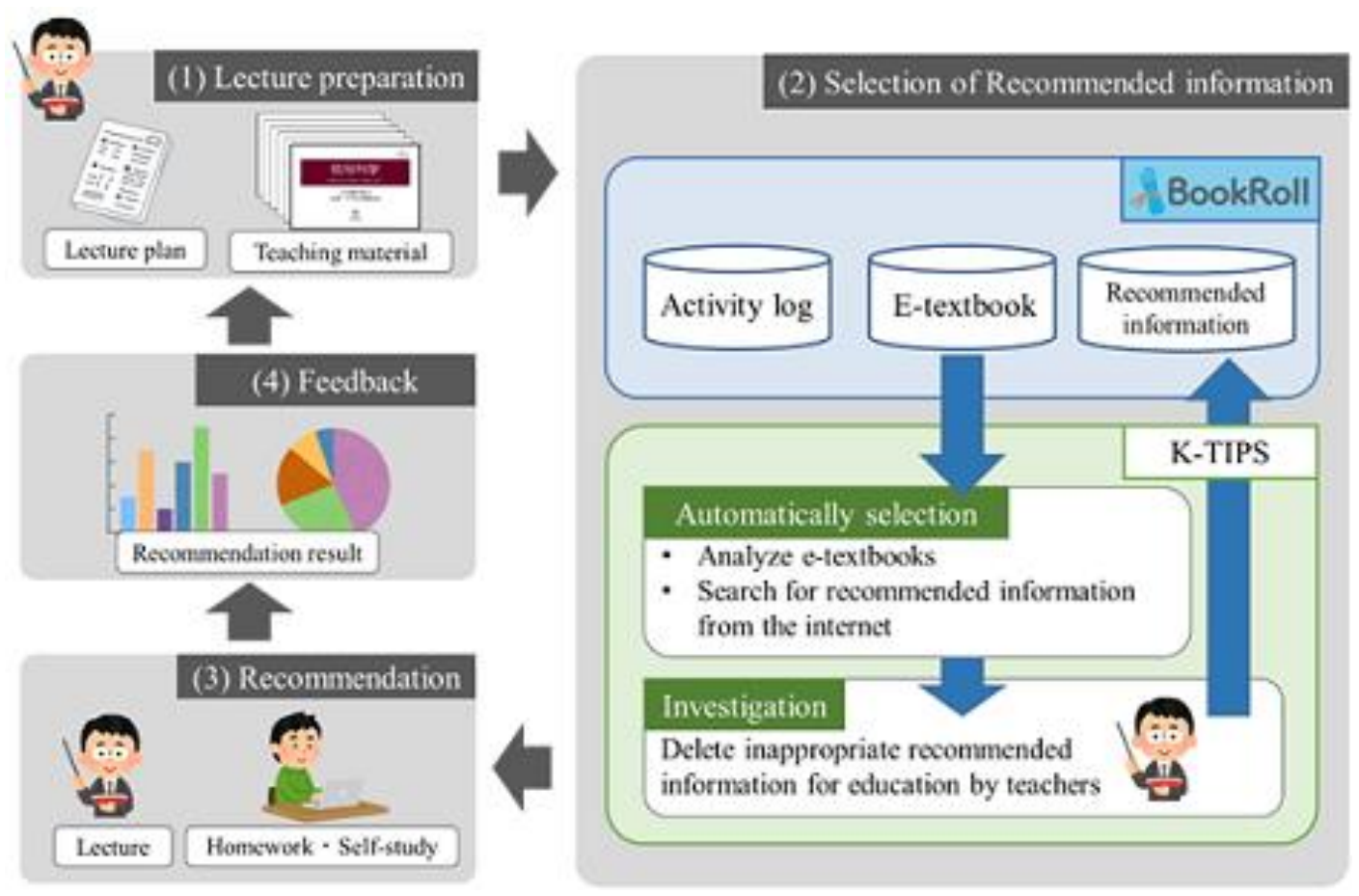

Figure 2. Overview of education improvement model 
A method to calculate recommended pieces of information in (2) is inspired by an existing study proposed by Nakayama et al (2019). According to the survey in the paper, the recommended items were mostly suitable for the content in the lecture materials. However, it sometimes contained inappropriate information for learning because the recommended items were automatically retrieved from the Internet and unilaterally provided to students. The problem is that it is not possible to know whether the recommended information is appropriate from an educational viewpoint or for individual learners. Therefore, it is necessary to have a mechanism for acquiring feedback from each user including the teachers and students. In order to solve this problem, in this study, a teacher intervenes in the selection of recommended information in (2). The system provides a list of candidates for recommended items on a web page, and the teacher investigates and decides whether each one is suitable for recommendation or not. We believe that teachers' intervention in selecting recommended information has several advantages for students and teachers, as listed below:

- We can prevent undesirable situations in which inappropriate or unnecessary information is recommended to students.

- The selection results by the teacher are helpful for the system to improve the automatic suggestion of recommended information.

The recommendation results for students are analyzed in (4) based on the student learning activity log (click on the link to the recommended information) in the e-Book system. The analysis results are feedback to teachers, students, and the system itself. Recommending information corresponding to each page of teaching material not only facilitates students' learning, but also allows teachers to understand the students' reaction to teaching materials used in lectures and proceed with a lecture. Therefore, teachers can use the analysis results as feedback from students to improve teaching materials and proceed with a lecture. The responses from students are also meaningful data that can be used to investigate whether recommended information was useful or not. The operation logs of clicking the recommended information indicates whether the student has interest in the recommended information. By collecting the access information of recommended information from each student, it will become possible to provide personalized recommended information according to the individual's learning level in the future. In this way, by examining and evaluating the recommended information from both teachers and students, it is possible to make high-quality recommendations, and further education improvement can be achieved. We will describe the system to realize this education improvement model in the following section.

\section{IMPLEMENTATION}

\subsection{System Configuration}

We use two systems to recommend information related to the teaching materials treated in lectures. One is an e-Book system called BookRoll (Ogata, 2015, 2017), the other is Knowledge extension based on Tailor-made Information Provision System (K-TIPS).

BookRoll is an e-Book viewer which has three databases. In each database, teaching materials that are e-textbook used in lectures, recommended information for each piece of teaching materials, and student's activity logs to e-Book system are stored. A teacher conducts a lecture using an e-textbook stored in the e-Book system and students learn by using it during and outside a lecture. Various functions are implemented in the e-Book system, and a recommendation function is one of them. Figure 3 shows the user interface of the e-Book viewer. Students can access the recommended items by clicking the upper right button of the viewer as necessary. In addition, each student can submit a response about whether he/she could understand the contents on the page or not, by clicking the green (understood) or red (not understood) button. When a student uses any of the functions of the e-Book system (such as open an e-textbook, go to the next page, highlight, click a link to a recommended article, etc.), learning activity logs are automatically recorded in the database. 


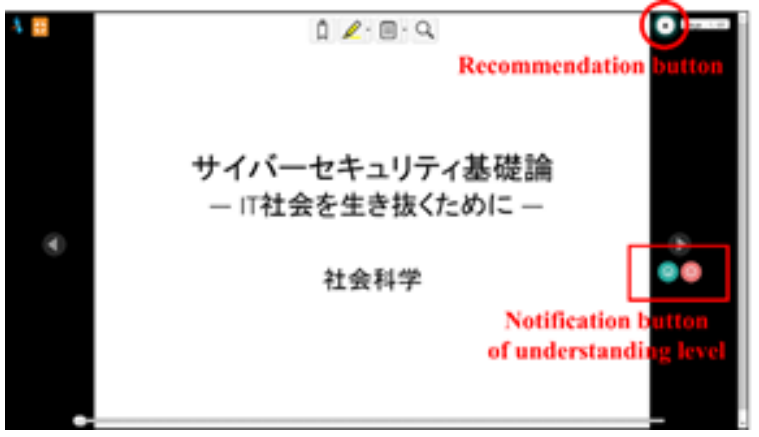

(A) Material viewer

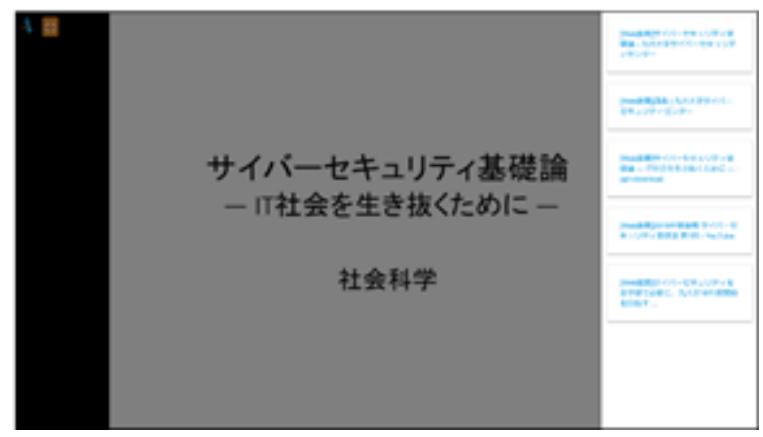

(B) Recommendation viewer

Figure 3. Viewer of e-Book system

The K-TIPS is a web application that displays candidates for recommended information, and allows teachers to select recommended information related to each page of teaching material. In this system, the teaching material designated by the teacher is analyzed, and the recommended information is automatically selected from the Internet and presented to the teacher. Then, the teacher investigates the candidates of recommended information and decides the information to be recommended to the student. Finally, it is registered in the e-Book system by the K-TIPS. The details of the web application are explained in the following sections.

\subsection{K-TIPS}

\subsubsection{Overview of K-TIPS}

In this research, as mentioned in section 2 , we believe that there are various advantages regarding the teacher's intervention in selecting recommended information. Therefore, we developed a web application for teachers to investigate the automatically selected recommended information from the Internet. The interface of the K-TIPS is shown in Figure 4.

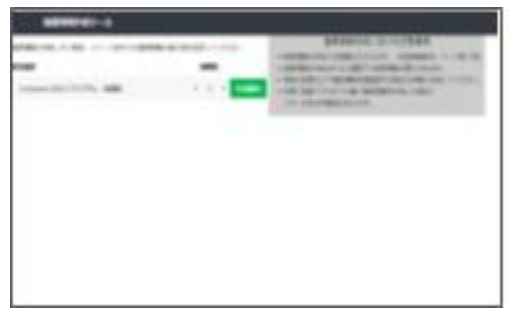

(A) Selection screen

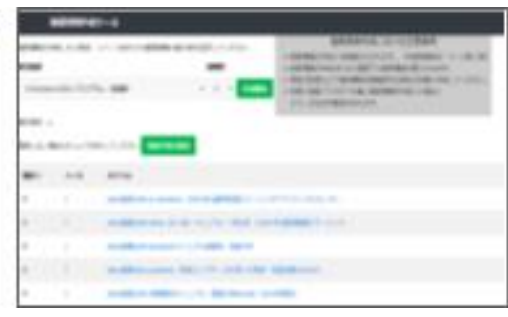

(B) Investigation screen

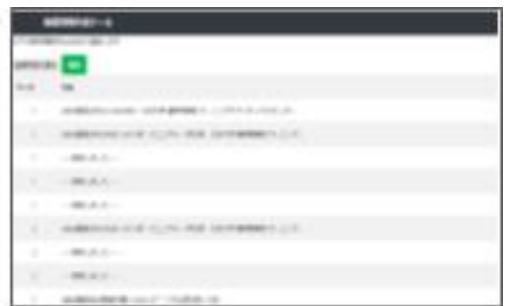

(C) Confirmation screen

Figure 4. Interface of K-TIPS

Figure 4-(A) is a web page for selecting lecture materials to be analyzed for calculating recommended information. In this page, the teacher specifies the maximum number of recommended items per page. When the specification is complete, they click a green button. Then, the recommended information for the teaching material is automatically selected from the Internet and displayed on the screen. The automatic selection method of recommended information is described in the next section.

Figure 4-(B) is a web page for investigation. On this page, two details about each recommended item are displayed in a table format: the page number recommended in the e-Book system and the title of the recommended item. In addition, a check box is displayed at the left end of the table. A link to the recommended information is attached to the displayed title, and the teacher can confirm the content of the recommended item by clicking the title. If the teacher judges that the content of the recommended information is not appropriate, they can uncheck the box. After completing the investigation of all candidates, they click the green registration confirmation button to proceed to the confirmation page. 
Figure 4-(C) is a web page to confirm the recommended information registered in the e-Book system. In this page, recommended items with boxes that are still checked after investigation are displayed, and recommended items with boxes that are unchecked are displayed as "deleted." The teacher verifies whether the recommended information to be registered is correct, and if there is some mistake, they can return to the investigation page again by clicking the return button. If there are no mistakes, the recommended information is registered in the e-Book system by clicking the registration button, and it is then recommended to the student.

This system stores a list of recommended items automatically selected from the Internet before investigation. Therefore, it is possible to analyze the recommended items deleted by teachers by comparing them with a list of recommended items registered in the e-Book system after investigation. The analysis results can be used to improve the accuracy of the automatic selection method of recommended information, and it is possible to support education and learning by providing recommendations that are more suitable for learning.

\subsubsection{Automatic Selection Method of Recommended Information}

In this section, we give an overview of the automatic selection method of recommended information for each page of a teaching material (Nakayama et al., 2019). In this method, to select recommended information, the method takes three analytical steps, as follows:

1. Word extraction from teaching material;

2. Calculation of the importance of extracted words;

3. Retrieval of recommended information from the Internet based on important words.

First, nouns in the teaching material are extracted. In the e-Book system, teaching materials are stored as PDFs, and text information about sentences in registered teaching materials is extracted from the PDFs automatically. Therefore, the text information can be utilized to extract sentences and words from the teaching material. In this research, we focus on Japanese teaching materials. In contrast to languages segmented by spaces such as English, Japanese is a non-segmented language. Therefore, it is necessary to divide the sentences to extract words. In this study, we apply MeCab morphological analysis (Kudo et al., 2004). Morphological analysis is the technique of dividing natural language into morphemes, the smallest units of words. In this method, proper nouns are extracted from a sentence through morphological analysis.

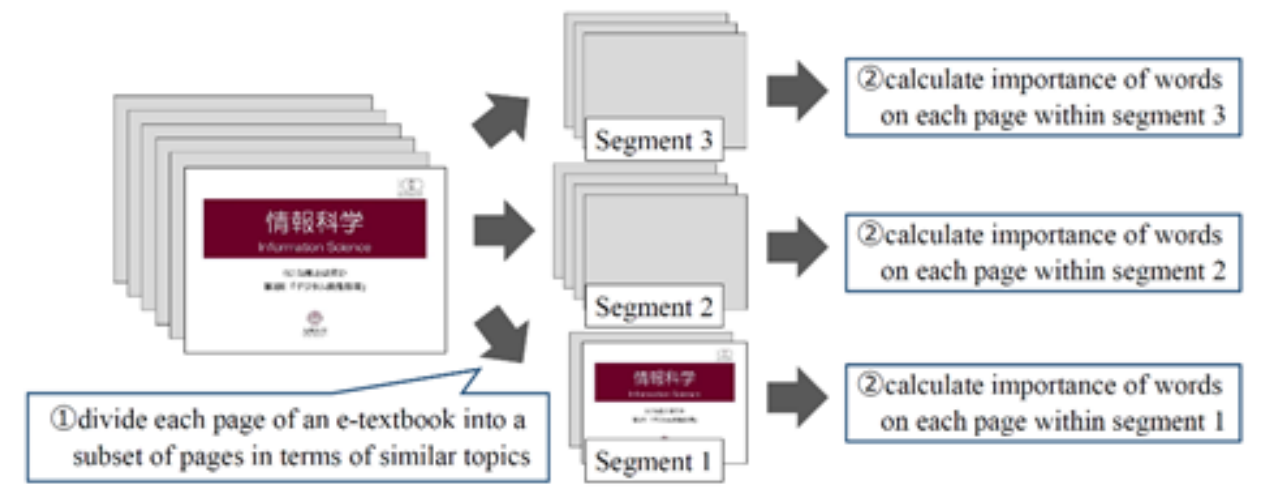

Figure 5. Overview of calculating importance of extracted words

Second, the importance of the extracted words on each page are estimated to select recommended information for each page of the teaching material. The estimation procedure involves two steps: 1) dividing each page of a teaching material into a subset of pages (refer to them as "segments") in terms of similar topics, and 2) calculating the importance of the words on each page within each segment. Figure 5 shows an overview of the process flow to estimate the importance of extracted words. In this method, whole pages of teaching material are divided into segments according to the topic of the contents because it may be considered that there is a difference in importance depending on the topic, even for the same word. After dividing into subsets of pages, the importance of each word is calculated for each segment. The importance of words in each segment is estimated using the TF-IDF method (Salton \& Buckley., 1988). The TF-IDF method is a method for evaluating the importance of words in a document using two indicators: term frequency (TF) and inverse document frequency (IDF). Teaching materials are divided into the title area and 
the body area. The title represents the contents of each page, and words in the title area seem to be important. Therefore, the importance of each word is calculated by weighing words in the title area to the importance of the TF-IDF method.

Third, recommended information is retrieved from the Internet based on the importance of the word. First, let the top $n$ words of importance in each page be the important words representing the content of the page. Next, the top $n$ words are used as the search query to retrieve related websites. Then, the top $m$ of the search results are selected to be the recommended items of information.

\subsection{Student Activity Log}

An analysis of recommendation results in (4) in section 2 is performed by analyzing student activity logs collected by the e-Book system. As mentioned above, various functions are implemented in the e-Book system. For example, adding a memo or a marker to a current page, evaluating whether a student understands the current page, accessing the current page's recommended information, etc. As shown in Figure 6, various activity logs of students using such functions can be collected.

An analysis of recommendation results uses logs for recommended information (a log with the operation name "CLICK_RECOMMENDATION" in Figure 6). From the log data of recommendations, it is possible to identify the user who accessed the recommended information, the page number of a teaching material, and which recommended information is accessed. Utilization of these learning activity logs has the possibility to improve education and learning in terms of following aspects:

- Update the teaching material by analyzing the number of clicks in each page of the teaching material.

- Determine the demand of each student for recommended information.

- Realize personalized recommendation.

Moreover, we believe that it is possible to analyze from other various viewpoints by utilizing the log data other than the recommendation. For example, it is possible to analyze the relationship between the reaction to the recommendation and the student's level of understanding by using the log indicating whether the student could understand the content of the current page (a log with the operation name "GETIT" or "NOTGETIT" in Figure 6). As a result, we believe that the recommendation results can be used to further improve education.

\begin{tabular}{|c|c|c|c|c|c|c|}
\hline User ID & e-book ID & e-book title & Page & Operation & Description & ... Time \\
\hline 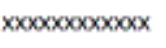 & 000000000 & D-06-画像 & 14 & CUICK_RECOMMENDATION & https: $/ / * * * * * * * * * * *$ & ... 2019-07-01 11:26:15 \\
\hline 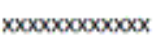 & 000000000 & D-06-函像 & 13 & NEXT & & ... 2019-07-01 11:26:17 \\
\hline 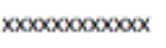 & 000000000 & D-06-画像 & 15 & NOTGETIT & & ... 2019-07-01 11:26:20 \\
\hline$x x 0 x x x 0 x x x x x$ & 000000000 & D-06-画像 & 16 & CLICK_RECOMMENDATION & https://*********** & ... 2019-07-01 11:26:25 \\
\hline 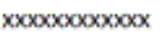 & 000000000 & D-06-画像 & 17 & GETIT & & ... 2019-07-01 11:26:31 \\
\hline
\end{tabular}

Figure 6. Samples of student activity logs stored in the e-Book system

\section{EXPERIMENTS}

We have been conducting experiments using the proposed system in multiple lectures for first- and second-year college students using the proposed system since April 2019. A total of 98 teaching materials used in 16 courses in the first semester of the 2019 school year at Kyushu University were used for the experiments and recommended information was provided to students by the end of July 2019. As a result of the recommendations, 1061 activity log data for recommendations were collected from 247 students. In this study, we have developed a web application for teachers to confirm the usage status of recommended information. Figure 7 is the display screen of the recommendation result. The teacher can confirm how many times the recommended items were clicked by students in each page. In the graph displayed, the x-axis is the page number of the teaching material, and the y-axis is the number of clicks for the recommended items of information. In addition, if the teacher moves the mouse cursor to the bar chart, its corresponding page is displayed below the graph. If the number of clicks for the recommended items is extremely high compared to other pages, it is taken into consideration that there may be a problem with the contents of the teaching 
materials or lectures. It has shown that the number of clicks on the relevant page may increase if the content of the teaching material is difficult or if there is a problem with the style of the lecture (Nakayama et al., 2019). In this way, utilizing the recommendation results can be used to improve education.

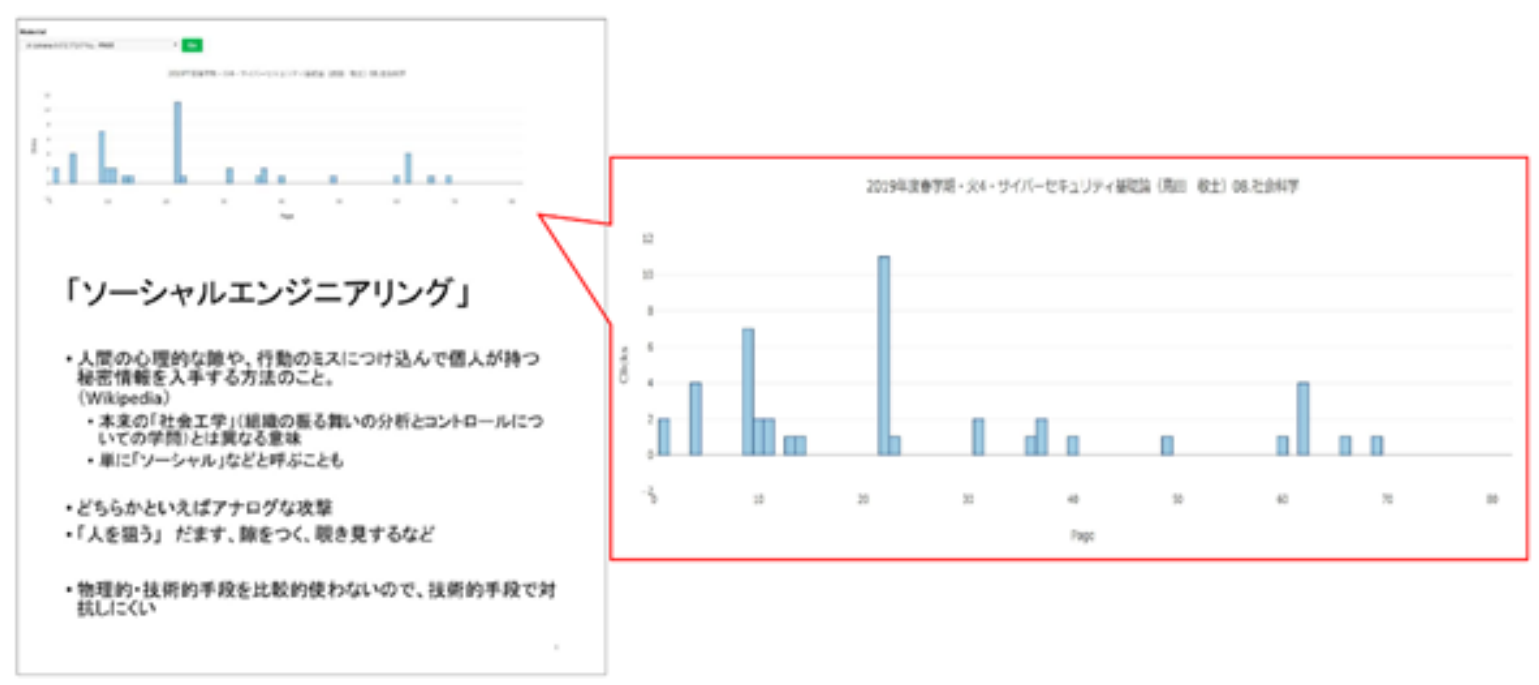

Figure 7. The display screen of the recommendation results

Also, investigation by teachers has been implemented since July 2019. By the end of July 2019, investigation was conducted by a total of four teachers for the recommended information on 42 teaching materials. As a result of the investigation, $1.4 \%$ of the recommended information selected automatically was judged as inappropriate or unnecessary for learning and was deleted by the teacher. We analyzed the titles and URLs of recommended information deleted by investigation. As a result, we saw that there were many words related to games (such as "games," "gamer," "game design," etc.) and words related to products (such as "Amazon," "product," and "shohin," which in Japanese means product, etc.) in the recommended information. The reason why the recommended information about the game was presented to the students is that an educational information engineering course included a class about game-based learning. In addition to this, many keywords such as "syllabus" and "yahoo" that were considered unnecessary for learning were confirmed. Based on the results of these investigations, we can implement high-quality recommendations for learning by designing a mechanism for automatically excluding information inappropriate for learning. In addition, we confirmed cases where all recommended items on some pages were deleted by investigation. It can be inferred that these were pages that did not require a recommendation (such as a page with notes about the lecture, etc.). It may be possible to identify pages that do not require a recommendation by using the investigation result.

\section{CONCLUSION}

In this paper, we proposed an education improvement model based on a system that recommends supplementary teaching materials related to teaching materials used in lectures. Then, we described the system for realizing the proposed education improvement and reported on the current status of recommendation experiments conducted in the university environment. We believe that the proposed system enables both teachers and students to improve their teaching and learning experiences through interaction with recommended information. A page in which many students used the recommended information suggests the possibility that the contents of the material are difficult to understand, so that a teacher will consider an update of the contents.

In future work, as mentioned above, we will work on the development of the method to calculate recommended information using the investigation result by the teachers and the learning support by the individual recommendation from the recommendation result to the student. In addition to these, we will also 
examine information recommendation for summarized teaching materials. The summarized teaching material is a collection of pages containing important content extracted from the teaching material (Shimada et al., 2017). The contents of the teaching material are automatically summarized to a small number of pages, so that only the essential points are given to learners. The combination of summarization technique and our recommender system will help learners understand the summary of lecture contents and present an opportunity for knowledge extension by using recommended information. We are sure that the collaboration with other digital technologies will support learning and teaching in a new digital educational era.

\section{ACKNOWLEDGEMENT}

This work was supported by JST AIP Grant Number JPMJCR19U1 and JSPS KAKENHI Grand Number JP18H04125, Japan.

\section{REFERENCES}

Chen, G. D. et al, 2007. Extending e-book with Contextual Knowledge Recommender for Reading Support on a Web-Based Learning System. International Journal on E-Learning, Vol. 6, No. 4, pp.605-622.

Chen, W. et al, 2014. A hybrid recommendation algorithm adapted in e-learning environments. World Wide Web, Vol. 17, No. 2, pp. 271-284.

Erdt, M. et al, 2015. Evaluating Recommender Systems for Technology Enhanced Learning: A Quantitative Survey, IEEE Transaction of Learning Technologies, Vol. 8, No. 4, pp. 326-344.

Flanagan, B. and Ogata, H., 2017. Integration of Learning Analytics Research and Production Systems While Protecting Privacy. In International Conference on Computers in Education (ICCE2017), pp. 333-338.

Kudo, T. et al, 2004. Applying conditional random fields to Japanese morphological analysis. In Proceedings of the 2004 conference on empirical methods in natural language processing.

Liang, G. et al, 2006. Courseware recommendation in e-learning system. In International Conference on Web-based Learning 2006, pp. 10-24.

Mbipom, B. et al, 2018. An e-learning recommender that helps learners find the right materials. The Eighth AAAI Symposium on Educational Advances in Artificial Intelligence 2018 (EAAI-18).

Nakayama, K. et al, 2019. Learning support system for providing page-wise recommendation in e-textbooks. In Society for Information Technology \& Teacher Education International Conference (SITE 2019), pp. 824-831.

Ogata, H. et al, 2015. E-Book-based learning analytics in university education. In International Conference on Computer in Education (ICCE 2015), pp. 401-406.

Salehi, M. et al, 2014. Personalized recommendation of learning material using sequential pattern mining and attribute based collaborative filtering. Education and Information Technologies, Vol. 19, No. 4, pp. 713-735.

Salton, G. and Buckley, C., 1988. Term-weighting approaches in automatic text retrieval. Information processing \& management, Vol. 24, No. 5, pp. 513-523.

Shimada, A. et al, 2017. Automatic Summarization of Lecture Slides for Enhanced Student Preview -Technical Report and User Study-. IEEE Transactions on Learning Technologies, Vol. 11, No. 2, pp. 165-178.

Tarus, J. K. et al, 2018. A hybrid recommender system for e-learning based on context awareness and sequential pattern mining. Soft Computing, Vol. 22, No. 8, pp. 2449-2461.

Yamada, M. et al, 2014. Collaborative filtering for expansion of learner's background knowledge in online language learning: does "top-down" processing improve vocabulary proficiency?. Educational Technology Research and Development, Vol. 62, No. 5, pp. 529-553. 